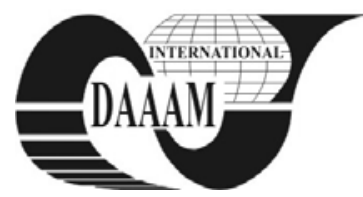

\title{
UNBALANCED MAGNETIC PULL AND ITS EFFECT ON DYNAMIC BEHAVIOR OF THE ROTOR WITH NONLINEAR ANISOTROPIC SUPPORT
}

\author{
DONAT, M[artin]
}

\begin{abstract}
The electromechanical interaction in rotating electric machines induces additional forces between the rotor and the stator. To study the influence of these forces on the dynamic behaviour of the rotor on nonlinear anisotropic supports, a simple computational model was developed. The aim of this study is to analyse the dynamic behaviour of the rotor of the three-phase generator with one pole pair, in dependence on the rotor angular frequency.
\end{abstract}

Key words: magnetic pull, rotor, structural anisotropy, amplitude-frequency response

\section{INTRODUCTION}

The magnetic fields in the air gap between the stator and the rotor, of electric rotating machines, induce electromagnetic forces, which act on the machines structure. If the thickness of the air gap is constant along whole circumference, the magnitude of the electromagnetic forces is zero. If the thickness of the air gap is not constant, the magnitude of the electromagnetic forces is nonzero and is called unbalanced magnetic pull (UMP). The effect of UMP on the rotor of electric rotating machines may has, under certain conditions, an important influence on the dynamic behaviour of the rotor, as shown for example in (Guo et al. 2002) or (Holopainen et al. 2002).

To study the influence of the UMP on the dynamic behaviour of the rotor on nonlinear anisotropic supports, a simple computational model was developed. This model is a combination of the de Laval rotor on nonlinear anisotropic supports and model of the electromagnetic forces, which operates between stator and the rotor, presented by (Guo et al. 2002).

\section{METHODS}

There is a problem of the circular vibration of the shaft. To solve this problem the approach based on transverse vibrations in two mutually perpendicular directions in the plane perpendicular to the axis of the rotor is used. Initial assumptions of this computational model are:

- The rotor is absolutely rigid.

- The rotor and the stator have an ideal cylindrical shape.

- The axis of the rotor is identical or parallel with the geometric axis of the stator.

- The supports of the shaft have structural anisotropy.

- Stiffness of the supports are nonlinearly dependent on the displacement.

- Damping forces are a function of the velocity only.

- Damping coefficients are constant over the entire operating range.

- UMP depends on displacement and time.
Computational model can by written as follows

$$
\begin{aligned}
& m \ddot{x}+b \dot{x}+k_{x} x=m a \Omega^{2} \cos (\Omega t)+F_{P x} \\
& m \ddot{y}+b \dot{y}+k_{y} y=m a \Omega^{2} \sin (\Omega t)-m g+F_{P y}
\end{aligned}
$$

Where $m$ is the mass of the rotor, $\Omega$ is the angular frequency of the rotor, $a$ is the mass eccentricity distance, $g$ is the gravitational acceleration, $t$ is time, $b_{x}, b_{y}$ are damping coefficients, $k_{x}, k_{y}$ are stiffness coefficients, $F_{P x}, F_{P y}$ are components of the UMP. The subscripts of the parameters $x$ and $y$ refer to respective dimensions to the axes of the coordinate system. $\ddot{x}, \ddot{y}$ are the accelerations, $\dot{x}, \dot{y}$ are the velocities, $x, y$ are dynamic displacements in directions of the coordinate systems.

Stiffness of both supports are the same and they are arranged in parallel mutually. According to (Genta 2005) the stiffness coefficients $k_{x}$ and $k_{y}$ of the system can be calculated as

$$
\begin{aligned}
& k_{x}=2 k_{s x}\left(1+\mu_{s x} x^{2}\right) \\
& k_{y}=2 k_{s y}\left(1+\mu_{s y} y^{2}\right)
\end{aligned}
$$

Where $k_{s x}, k_{s y}$ are the base stiffness coefficients of the supports, $\mu_{s x}, \mu_{s y}$ are coefficients of nonlinearity and $x, y$ are displacements of the centre of gravity of the shaft in directions of the coordinate systems.

According to (Gash \& Pfützner 1975) the damping coefficients of the supports are arranged with the damping coefficient of the surroundings in parallel. The damping coefficients $b_{x}$ and $b_{y}$ are thus calculated as

$$
\begin{aligned}
& b_{x}=2 b_{\text {sx }}+b_{\text {sur }} \\
& b_{y}=2 b_{\text {sy }}+b_{\text {sur }}
\end{aligned}
$$

Where $b_{\text {sur }}$ is the damping coefficient of the surroundings and $b_{s x}, b_{s y}$ are the damping coefficients of the supports.

The components of the UMP, forces $F_{P x}$ and $F_{P y}$ are calculated from equations (6) and (7), which were published by (Guo et al. 2002).

$$
\begin{aligned}
& F_{P x}=f_{1} \cos (\Omega t)+f_{2} \cos (2 \omega t-\Omega t)+f_{3} \cos (2 \omega t-3 \Omega t)(6) \\
& F_{P y}=f_{1} \sin (\Omega t)+f_{2} \sin (2 \omega t-\Omega t)-f_{3} \sin (2 \omega t-3 \Omega t)(7)
\end{aligned}
$$

Where $t$ is time, $\Omega$ is the rotor angular frequency, $\omega$ is the frequency of the electricity supplied. Coefficients $f_{1}, f_{2}$ and $f_{3}$ are calculated from equation (8), (9) and (10).

$$
f_{1} \approx \frac{R L \pi \mu_{0} F_{j}^{2}}{\delta_{0}^{2}}\left(\frac{1}{2} \varepsilon+\frac{5}{8} \varepsilon^{3}\right)
$$




$$
\begin{gathered}
f_{2} \approx \frac{R L \pi \mu_{0} F_{j}^{2}}{\delta_{0}^{2}}\left(\frac{1}{4} \varepsilon+\frac{5}{16} \varepsilon^{3}\right) \\
f_{3} \approx \frac{R L \pi \mu_{0} F_{j}^{2}}{8 \delta_{0}^{2}} \varepsilon^{3}
\end{gathered}
$$

Where $R$ is the radius of the generator rotor, $L$ is the length of the generator rotor, $\mu_{0}$ is the air permeance, $F_{j}$ is the fundamental magneto motive force amplitude of the rotor exciting current, $\delta_{0}$ is the mean air gap length. The parameter $\varepsilon$ is calculated from equation (11)

$$
\varepsilon=\frac{\sqrt{\left(x+x_{\text {stat }}\right)^{2}+\left(y+y_{\text {stat }}\right)^{2}}}{\delta_{0}}
$$

Where $x, y$ are dynamic displacements and $x_{\text {stat }}, y_{\text {stat }}$ are static eccentricity.

\section{RESULTS}

Dynamic system (1) was solved numerically by DormandPrice method $(5,4)$, which belongs to the Runge-Kutta methods family. Descriptions of this method can be found for example in (Čermák 2010). Parameters used for the calculations are: $m=18,15 \mathrm{~kg} \quad b_{s x}=900 \frac{\mathrm{Ns}}{\mathrm{m}} \quad \mu_{s x}=2 \cdot 10^{7} \frac{1}{\mathrm{~m}^{2}}$ $R=0,059 \mathrm{~m} \quad b_{s y}=1000 \frac{\mathrm{Ns}}{\mathrm{m}} \quad \mu_{s y}=2 \cdot 10^{7} \frac{1}{\mathrm{~m}^{2}}$ $L=0,1551 \mathrm{~m} \quad k_{s x}=2,5 \cdot 10^{6} \frac{\mathrm{N}}{\mathrm{m}} \quad \mu_{0}=4 \cdot \pi \cdot 10^{-7} \frac{\mathrm{Vs}}{\mathrm{Am}}$ $a=5 \cdot 10^{-5} \mathrm{~m} \quad k_{s y}=5 \cdot 10^{6} \frac{\mathrm{N}}{\mathrm{m}} \quad F_{j}=684 \mathrm{~A}$ $\delta_{0}=2,2 \cdot 10^{-3} \mathrm{~m} \quad \omega=50 \mathrm{~Hz} \quad \mathrm{~m} \quad x_{\text {stat }}=0 ; 5 \cdot 10^{-4} \mathrm{~m}$ $b_{\text {sur }}=819 \frac{\mathrm{Ns}}{\mathrm{m}} \quad g=9,81 \frac{\mathrm{m}}{\mathrm{s}^{2}} \quad y_{\text {stat }}=0 \mathrm{~m}$

In Figures 1. and 2. are amplitude-frequency responses of the rotor. Three cases were studied:

a) Rotor without UMP and its axis was the same as the axis of the stator at initial state $\left(x_{\text {stat }}=0 \mathrm{~mm}\right)$.

b) Rotor with UMP and its axis was the same as the axis of the stator at initial state $\left(x_{\text {stat }}=0 \mathrm{~mm}\right)$.

c) Rotor with UMP and its axis was parallel to the axis of the stator with static eccentricity $x_{\text {stat }}=0,5 \mathrm{~mm}$ at initial state.

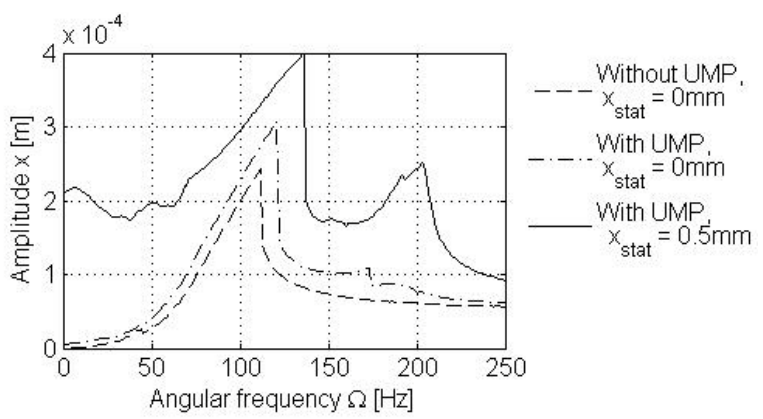

Fig. 1. Amplitude-frequency response of the rotor in the direction of the $\mathrm{x}$-axis

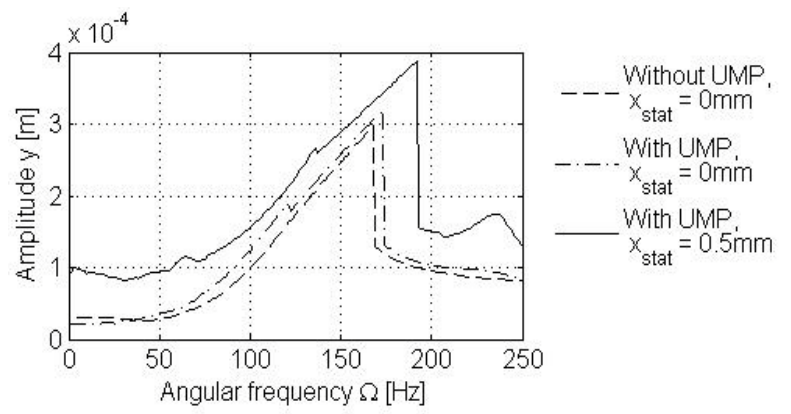

Fig. 2.Amplitude-frequency response of the rotor in the direction of the $y$-axis
If we compare the amplitude-frequency responses in case a) and b), in the Figure 1. or 2., we can see that UMP in electric machines causes larger displacements of the rotor, moreover UMP causes coupled vibration of the rotor, because in case b) the rotor oscillates significantly not only in the natural frequency of vibration in the direction of the $\mathrm{x}$-axis, but also significantly oscillates in the natural frequency of vibration in the direction of the y-axis. If we compare the amplitudefrequency responses in cases b) and c) in the Figure 1. or 2., we can see that, in case c), the rotor oscillates considerably not only when the angular frequency of the rotor is close to the natural frequencies, but also the rotor oscillates considerably when the angular frequency is close to $200 \mathrm{~Hz}$ (in direction $\mathrm{x}$ ) and $235 \mathrm{~Hz}$ (in direction y). The explanation of this phenomenon is evident from the equations (6) and (7). For example, if the angular frequency of the rotor is $\Omega=235 \mathrm{~Hz}$, the frequency of the second components of the equations (6) and (7) is close to the natural frequency of the rotor in the direction of the $y$-axis, see Figure 3 . Notice, backbone in this figure is computed without considering UMP.

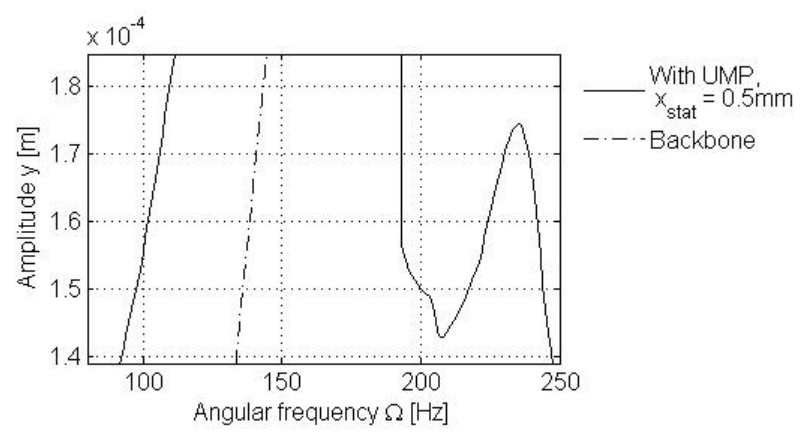

Fig. 3. Detail of the amplitude-frequency response of the rotor in the direction of the y-axis

\section{CONCLUSIONS}

The aim of this study was to create a computational model for studying the influence of nonlinear UMP on dynamical behaviour of the rotor supported by nonlinear bearings with structural anisotropy. The obtained results confirmed that UMP can significantly affect the vibration of the rotor.

UMP is reflected in the equation of motion as an additional stiffness, so the next step will be to evaluate the impact of the UMP on natural frequencies of the rotor. These results should be presented in the conference.

\section{ACKNOWLEDGEMENT}

This paper was written with support by the EU, project no.: CZ.1.07/2.4.00/12.0030

\section{REFERENCES}

Čermák, L. (2010). Numerical methods II differential equations, Academic publishing CERM, ISBN 978-80-2144110-1, Brno

Gash, R. \& Pfützner, H. (1980). Rotor dynamics, SNTL, Praha

Genta, G. (2005). Dynamics of rotating systems, Springer, ISBN 0-387-20936-0, Torino

Guo, D.; Chu, F. \& Chen, D. (2002). The unbalanced magnetic pull and its effects on vibration in three-phase generator with eccentric rotor. Journal of Sound and Vibration, Vol. 254, No. 297-312

Holopainen, T. P.; Tenhunen, A. \& Arkkio, A. (2002). Electromagnetic circulatory forces and rotordynamic instability in electric machines, Proceedings of the 6th International Conference on Rotor Dynamics, Australia, ISBN 0-7344-1963-1, Hahn, E. J. \& Randall, R. B. (Ed), pp. 446-463, University of New South Wales Printing Services Sydney 